\title{
Gestión en la educación, perspectivas renovadoras ${ }^{1}$
}

https://doi.org/10.21830/9789585241459.04

\author{
Leidy Johana Cabrera Cabrera ${ }^{2}$ \\ Escuela Militar de Cadetes "General José María Córdova” \\ María Antonieta Corcione Nieto ${ }^{3}$ \\ Escuela de Aviación del Ejército Nacional \\ John Cristhian Fernández Lizarazo ${ }^{4}$ \\ Universidad de La Salle \\ John Alexander León Torres 5 \\ Escuela de Armas Combinadas del Ejército
}

\section{Resumen}

Antecedentes: la gestión en la educación se ha reducido tradicionalmente a aspectos de tipo administrativo que responden a directrices establecidas institucionalmente y a tendencias neoliberales. Sin embargo, abordar la gestión en la educación de manera integral implica consi-

1 Capítulo de libro que parte de los resultados de los proyectos de investigación Geopolítica del Conocimiento sobre Conflicto y Paz en Colombia: Aportes, Debates y Tendencias, del Grupo de Investigación en Ciencias Militares de la Escuela Militar de Cadetes "General José María Córdova” (ESMIC), registrado con el código COL0082556 de Minciencias, y del proyecto El Quehacer de las Mujeres en el Ejército Nacional de Colombia. Una aproximación desde las Experiencias Femeninas, del Grupo de Investigación en Aviación Militar, de la Escuela de Aviación del Ejército (Esave), registrado con el código COL0077618 de Minciencias. Los puntos de vista y los resultados de este trabajo pertenecen a los autores y no reflejan necesariamente los de las instituciones participantes.

2 Magíster y especialista en Docencia e Investigación Universitaria de la Universidad Sergio Arboleda. Trabajadora social de la Universidad de La Salle e investigadora del Grupo de Investigación en Ciencias Militares de la ESMIC. Orcid: https://orcid.org/0000-0002-6398-6933. Contacto: leidy.cabrera@esmic.edu.co

$3 \mathrm{PhD}$ en Antropología de la Universidad de los Andes y magíster en Biología Humana de la Universidad Autónoma de Barcelona. Investigadora del Grupo de Investigación en Aviación Militar de la Escuela de Aviación del Ejército. Orcid: https://orcid.org/0000-0002-3509-5519. Contacto: mariacorcionenieto@cedoc.edu.co

$4 \mathrm{PhD}$ y magíster en Ciencias Agrarias de la Universidad Nacional de Colombia y licenciado en Biología de la Universidad Distrital Francisco José de Caldas. Director del programa de Ingeniería Agronómica de la Universidad de La Salle. Orcid: https://orcid.org/0000-0001-9015-7404. Contacto: jhfernandez@lasalle.edu.co

5 Teniente Coronel del Ejército Nacional de Colombia. Inspector de Estudios de la Escuela de Armas Combinadas del Ejército. Candidato a magister en educación de la Universidad Militar Nueva Granada. Especialización en seguridad y defensa nacionales de la Escuela Superior de Guerra "General Rafael Reyes Prieto". Especialización en docencia Universitaria del Centro de Educación Militar. Profesional en ciencias militares de la Escuela Militar de Cadetes "General José María Córdova”. Profesional en relaciones internacionales y estudios políticos de la Universidad Militar Nueva Granada. Orcid: https://orcid.org/0000-0001-6279-8351. Contacto: johnleontorres@cedoc.edu.co 
derar una perspectiva denominada renovadora, la cual se caracteriza por ubicar al ser humano en el centro de los procesos educativos y como actor determinante en su desarrollo. Objetivo: en este capítulo se muestran los aportes, para el avance hacia este tipo de gestión en la educación militar, propuestos por Benno Sander (1991) y Juan Casassus (2001), quienes se han enfocado en superar la visión instrumental de la gestión en el campo educativo. Materiales y métodos: se trata de un estudio documental que permite esbozar lineamientos importantes que pueden retomarse para la formación militar en su avance hacia una formación integral. Resultados: por medio de la generación de categorías deductivas (descentralización educativa, enfoque dialéctico y cultura institucional) se muestra que esta última orienta los procesos académicos, administrativos e institucionales que se configuran como ejes determinantes en el escenario de formación militar. Conclusiones: la perspectiva humanista y sus enfoques sociales, renovadores y críticos, permite una amplía reflexión sobre los retos que actualmente tiene la gestión en la educación militar, lo cual requiere de la generación de espacios de diálogo permanentes.

Palabras clave: administración; educación; enfoques; formación militar; gestión en la educación; pedagogía.

\section{Introducción}

Desde finales del siglo pasado, las políticas educativas en Colombia han adquirido un tenor neoliberalista. Según Estrada (2002), estas transformaciones en materia educativa estuvieron enmarcadas en dos dimensiones; la primera relacionada con una cultura y una pedagogía del (y para el) mercado, de exaltación del rendimiento individual, el productivismo y la competencia; y la segunda relacionada con la eficiencia en términos de financiación, cobertura y calidad. Lo que sugiere, por una parte, una mejora en la gestión en la educación y, por la otra, el replanteamiento de la visión administrativa no humanista por cuenta de la naturaleza misma de la realidad de cada institución.

Como resultado, ambas dimensiones han configurado problemas concretos de la gestión educativa que se explicitan en aspectos como las diferentes comprensiones de los directivos docentes hacia la gestión en las instituciones educativas, ya sea por rechazos, desconocimiento, desinterés o desconexión con la labor educativa y pedagógica; la identificación de la gestión con funciones administrativas, normativas e instrumentalistas, que no son compatibles con la creatividad de los procesos de enseñanza y de aprendizaje; la separación de las concepciones, modelos, procesos pedagógicos y académicos de los de gestión educativa, que impiden que esta sea el apoyo real a la educación y la cultura institucional, expresada en efectos de burocratización, normatización, instrumentalización, y funciones de dirección y control, 
aisladas de la participación, el trabajo académico y los proyectos investigativos; la centralización de la gestión educativa, entendida como administración y dirección, concentrada en los directivos, en resultados medibles y no en los colectivos, en la comunidad académica ni en los procesos educativos; la identificación de la gestión con orientaciones centralizadas desde las políticas educativas y organismos superiores, mas no en los proyectos internos y de las características de los contextos y culturas institucionales para desarrollar propuestas propias a los problemas de cada ambiente institucional.

Ante este panorama, se considera importante abordar el fundamento teórico-conceptual de la gestión en la educación desde una perspectiva humanista y sus enfoques sociales, renovadores y críticos. En este sentido, se revisaron algunas posturas sobre la forma como se entiende al ser humano en su relación con la gestión educativa, lo que permitió avanzar en un primer análisis que podrá retomarse en el proceso actual de consolidación educativa e investigativa en el escenario militar.

Pensar la educación científica y la cultura investigativa desde la gestión en las instituciones educativas como un continuo relacionado ha sido un tema poco desarrollado en escenarios investigativos. Por lo tanto, los postulados que se presentan en este capítulo parten de la construcción de una perspectiva que permite evitar las miradas reduccionistas y dicotómicas del ser humano para ubicarlo en el centro de la educación y de la gestión. Esta perspectiva ha tomado fuerza en los diversos ámbitos educativos de América Latina, por lo que requiere asumir sus desafíos desde las diversas áreas de formación. Siendo así, el análisis que se presenta a continuación constituye un aporte a la formación del profesional en ciencias militares de Colombia.

El profesional en ciencias militares de Colombia se configura como un profesional con competencias para desempeñarse como comandante de pelotón, hábil en el desarrollo de misiones tácticas terrestres e instructor de tropas. Además, participa en procesos educativos en las escuelas de capacitación y unidades de entrenamiento (ESMIC, 2019).

Este capítulo se basa en los resultados obtenidos en el trabajo de grado de maestría en docencia e investigación universitaria titulado Principales enfoques y tendencias sobre la gestión en la educación en docentes de educación media y básica que cursan estudios de maestría en docencia e investigación universitaria en la Universidad Sergio Arboleda (Cabrera, 2016). Ese documento tiene cuatro apartados de desarrollo conceptual que definen los elementos básicos para comprender la gestión en la educación, los cuales permitieron generar categorías integradoras sobre esta. 
En la primera parte, se exponen las concepciones sobre enfoques y gestión educativa, así como algunos hitos históricos de la administración que han dado paso a diferentes conceptos y perspectivas propios de la disciplina. De estos aspectos se profundiza en aquellos que han aportado al desarrollo de la gestión y posteriormente a la gestión en la educación.

La segunda parte describe, desde los planeamientos de Omar Aktouf (2001), de la escuela de Montreal, y Le Mouel, de la escuela francesa, una perspectiva de la administración y la gestión a partir de un enfoque humanista de los estudios críticos de la gestión.

Posteriormente, se analizan los postulados sobre gestión en la educación de Benno Sander (1994) y Juan Casassus (1999). Estos autores han sido seleccionados por la coherencia en sus planteamientos y enfoques de tipo social-humanista y crítico. La propuesta de Sander y Casassus, por una parte, rechaza lo instrumental, el mecanicismo, la separación del conocimiento y de la naturaleza humana como elementos únicos y centrales en la gestión, y por otra parte, plantea un abordaje de la gestión en la educación no como administración, sino en su relación con la educación, la pedagogía y la cultura.

Finalmente se reconocen los enfoques renovadores de la gestión en la educación desde una perspectiva humanista, como elementos de reflexión y posteriores análisis frente a la formación de futuros militares, toda vez que la formación militar pasa por un proceso de consolidación permanente en el escenario académico, científico y práctico.

El desarrollo conceptual de este capítulo se basó en la propuesta metodológica y en algunos resultados del estudio que hizo el Grupo de Investigación en Autoevaluación Institucional de la Universidad Pedagógica (2014-2015) denominado "Enfoques y tendencias epistemológicas y teóricas de la Gestión en Educación en Colombia desde mediados de los años noventa hasta la actualidad". De este estudio se retomaron dos de los autores determinantes para la gestión en la educación en Colombia (Benno Sander y Juan Casassus), los cuales mantienen una perspectiva humanista y renovadora en sus actuales debates sobre la gestión en la educación.

\section{Enfoques y tendencias en la gestión educativa}

Una de las premisas sobre el enfoque, enuncia que cuando este se universaliza y tiene seguidores puede llegar a constituirse en escuela (Alvarado, 1999). En esta frase se evidencia la fuerza que puede tener un enfoque en la construcción de 
conocimiento, puesto que implica una forma de entender, analizar o estudiar algún fenómeno o hecho social.

Para Sander (1996a), los enfoques en la gestión educativa están basados en descubrir, dirigir, proyectar y comprender los puntos esenciales de un asunto o problema educativo para tratarlo acertadamente de una manera flexible, ya que se puede replantear para así dar origen a modelos y corrientes pedagógicas.

Históricamente, de acuerdo con Sander (1996b), han existido cinco enfoques analíticos que se han reflejado en la historia de la administración de la educación en América Latina: (1) enfoque jurídico, relacionado con una etapa normativa en la que prevalece el derecho administrativo romano y el pensamiento deductivo; (2) enfoque tecnocrático, que enfatiza en la adopción de perspectivas y soluciones organizacionales desde el pragmatismo instrumental; (3) enfoque conductista, en el que se rescata la dimensión humana de la administración y de la teoría de los sistemas como instrumento analítico para estudiar la situación y el funcionamiento de los sistemas educativos; (4) enfoque desarrollista, que se establece en la posguerra y presenta una perspectiva de la educación dedicada específicamente a la gestión de los programas de desarrollo para lograr sus objetivos económicos y sociales, y (5) enfoque sociológico, que presenta las contribuciones conceptuales y analíticas de las ciencias sociales aplicadas de los autores latinoamericanos de las últimas décadas.

Este esquema analítico sugiere que los enfoques del pensamiento administrativo en la educación latinoamericana resultaron de un proceso de construcción, desconstrucción y reconstrucción permanente a lo largo de la historia de las instituciones políticas y sociales. Esto implicó, además, un permanente desarrollo interdisciplinar de pensadores de diversas líneas de conocimiento que generaron interesantes construcciones a partir de una realidad social y política.

Con estos enfoques no se pretende unificar el concepto de gestión ni generalizarlo. Así, la definición de enfoque la referimos en este capítulo como el modo de integrar diversos factores sociales, políticos, económicos y culturales presentes en la dinámica de un contexto para estudiarlo y descubrir la mejor forma de abordar un fenómeno, para este caso la gestión en la educación. Cabe señalar que es un concepto flexible y dinámico que está en permanente construcción desde las mismas realidades y subjetividades.

Asimismo, Sander (2002) establece que la tendencia es una orientación de ideologías que se asume y que permite generar posturas diversas frente a un determinado asunto. Para el caso de la gestión en la educación se presentan nuevas tendencias basadas en la democracia y la calidad (Sander, 1996b). Por su parte, Botero (2009) explica cinco tendencias que se han presentado en la educación superior 
del siglo XXI, en las cuales se le atribuye un escenario importante a la gestión en la educación para su revisión y estudio: (1) autonomía, que presupone actuar sobre asuntos prioritarios en el mejoramiento y transformación de los procesos educativos en las instituciones; (2) democracia, que asume que la ciencia no es patrimonio de una élite, ni de lo que se llamó la aristocracia de la inteligencia, sino de toda la sociedad, más aún de la humanidad; (3) calidad, que busca formar ciudadanos capaces de comprender las complejas interrelaciones existentes entre ciencia, tecnología y los ámbitos social, económico, político y cultural, con el fin de que en el futuro, cualquiera que sea su esfera de actividad, cuenten con los instrumentos imprescindibles para participar en la toma de decisiones que contribuya a construir una sociedad democrática basada en el desarrollo científico y tecnológico; (4) formación integral, que se refiere a convertir las instituciones en generadoras de un clima que posibilite el ejercicio de valores en la cotidianidad, en las aulas, en los patios y en las instancias de decisión; (5) surgimiento de la universidad virtual, que requiere redefinir el concepto actual de universidad, explorar y construir un concepto de educación virtual, su significado y objetivos.

Como se denota, las tendencias sobre gestión en la educación muestran una evolución continua en la búsqueda de su comprensión. Por tal razón, representan un reto importante para este estudio, en la medida en que aportan elementos de mayor profundidad en el análisis de la gestión en la educación.

\section{La administración, un paso necesario para hablar de gestión}

Sin que el propósito de este capítulo sea profundizar en el desarrollo histórico de la administración, es importante comprender los hitos que la han consolidado como la conocemos actualmente, así como reconocer sus principales ejes de trabajo, los cuales están asociados a diferentes contextos y culturas en los que se ha desarrollado.

Las pautas en administración se dan con la división del trabajo. Ya para el período medieval, la organización feudal reafirma el concepto de autoridad y de equilibrio entre la autoridad central y la autonomía local; los mercaderes de Venecia (1418-1449), con sus propuestas de intercambios de productos, así como los escritores del siglo XVI Tomás Moro y Nicolás Maquiavelo, que expusieron importantes conceptos sobre el tema de administración, fueron un primer esbozo de los principales referentes en este campo (Monroy, 2002). 
En siglo XIX, Weber (1947) consideró a la organización del trabajo como un fenómeno burocrático y también como un proceso racionalizador orientado a ajustar los fines con los medios. Por su parte, Follett (1965), propuso que las organizaciones funcionaran con el principio del poder "con" y no del poder "sobre"; así reconoció la naturaleza holística de la comunidad y desarrolló aún más la idea de relaciones recíprocas, lo cual permitiría el entendimiento de los aspectos dinámicos del individuo en su relación con otros. Follett (1965) también defendió el principio de integración y resaltó la importancia de "compartir el poder". Esto implica que quienes conforman la organización deben integrarse mediante la conciliación y la adaptación más o menos racional, es decir, voluntaria (Follet, 1965).

Es a finales del siglo XIX y a comienzos del siglo XX cuando James Steuart, Adam Smith, Richard Arkwright y otros propusieron nuevos conceptos sobre la producción, la distribución, el comercio, la planificación y la administración, que se convirtieron en un marco de referencia para la propuesta de Taylor (2000), seguida por la de Fayol (2000) en la primera mitad del siglo XX (Monroy, 2002). Los principios de la administración científica que propusieron ambos autores introdujeron temas como la creación de incentivos para aumentar el logro y el resultado, los estándares, el control de tiempos y movimientos, la ingeniería de métodos, la selección científica de personal, la separación del trabajo intelectual y material, lo cual permitía proyectar a la organización de manera vertical para lograr administrarla y maximizar el trabajo. De este modo se abrió el camino para el estudio epistemológico y científico de lo que hoy se conoce como administración y que sigue siendo de gran relevancia cuando de eficacia y productividad se trata (Münch, 2007).

Asimismo, se encuentra la escuela humanística, cuyos mayores exponentes son Mayo, Lewin, Roethlisberger, Dickson, McGregor y Maslow. El principal postulado de la escuela humanista consiste en humanizar y democratizar la administración, liberarla de los conceptos rígidos y mecanicistas del enfoque clásico y adecuarla a los nuevos patrones de vida del pueblo norteamericano. En este sentido, la escuela humanista se convirtió en un movimiento típicamente norteamericano dirigido hacia la democratización de los conceptos administrativos y al desarrollo de las llamadas ciencias humanas, principalmente la psicología y la sociología, como también su creciente influencia intelectual y sus primeros intentos de aplicación a la organización industrial.

Dentro de esta escuela se destacaron posteriormente algunos sociólogos, como es el caso de Fayol (2000), quien racionaliza la función del trabajo. Luego sobresale Mayo (2004) quien hace un análisis sobre los problemas humanos de la sociedad industrial. Este autor enfatizó su trabajo en las motivaciones económicas en el 
proceso laboral, lo que originó la escuela de relaciones humanas y su énfasis en las personas. La propuesta de Mayo (2004), conocida como teoría organizacional humanista, distingue diversas nominaciones que responden a los múltiples subenfoques que se derivan de ella, entre ellos la teoría del comportamiento y la teoría del desarrollo organizacional. Estos enfoques contribuyeron posteriormente a que la corriente humanística evolucionara, de manera que sus postulados han sido determinantes en el éxito de cualquier organización productiva (Stein, 1999).

Es importante considerar que la escuela humanista de la administración se encontraba enmarcada por la depresión económica mundial y surgió en oposición a la escuela clásica, de manera que postuló nuevas competencias de los directivos acordes con la expansión y modernización de las organizaciones. Igualmente, Lewin (1951) tuvo gran impacto sobre la gestión moderna, sus postulados son ampliamente citados y sus alumnos fueron los principales promotores de la escuela de relaciones humanas, gestión de personal y psicología industrial. El ingenio innovador de este investigador en el campo experimental proporcionó un punto de vista científico, base para muchos de los conceptos actuales en la disciplina de la gestión (Wolf, 1973). Entre sus principales contribuciones a la teoría de la gestión se puede referenciar los conceptos de la teoría de campo; la investigación en acción, que hace referencia al servicio al cliente; el estudio de la dinámica de grupos y aspectos de sensibilidad de las técnicas de formación. A su vez centró su atención en el liderazgo autoritario y en especial en los estilos del liderazgo democrático, que son los que determinan el comportamiento del grupo y no las diferencias individuales. También hizo aportes a la teoría gerencial. Se considera precursor de la administración y del trabajo en equipo como condición primordial de la administración. También privilegió la importancia del trabajo y las relaciones interpersonales.

En la década de los años cincuenta, Haire, Maslow y Whyte realizaron diferentes aportes, entre los que se destacan los experimentos sobre la actitud de los empleados frente a sus labores; es decir, se enfocaron en la personalidad de los empleados y en la capacidad de liderazgo de cada uno según su rol u oficio en la empresa. Maslow propuso lo que aún se denomina "la pirámide de Maslow" o "jerarquía de las necesidades humanas", que consta de cinco niveles y su idea principal es que las necesidades más altas ocupan la atención solo cuando se han satisfecho necesidades inferiores. Es decir que el empleado solo se preocupa de temas relacionados con la autorrealización si está seguro de que tiene un trabajo estable, comida asegurada y un entorno social que lo acepta (Boeree, 2003).

Por su parte, McGregor (1994), quien trató en sus estudios el lado humano de las organizaciones, introdujo la nueva tendencia en todo el campo de la admi- 
nistración, en las nuevas áreas del comportamiento y el desarrollo organizacional, haciendo énfasis en el ser humano y el papel de las personas en la sociedad industrial. En 1960 presentó dos hipótesis sobre el comportamiento humano: la teoría $\mathrm{X}$ desde el punto de vista tradicional sobre la dirección y el control; y la teoría $\mathrm{Y}$, como alternativa para integrar los objetivos individuales con los de la organización. Asimismo, manifestó preocupación trascendental por la dignidad, el valor y el desarrollo del individuo, y abordó la situación del conflicto entre las necesidades individuales, a través de relaciones interpersonales efectivas, entre superiores y subordinadas. De igual manera, postuló el concepto de influencia, basado no en la coerción sino en la apertura, en la confrontación y en el proceso de ventilar las diferencias.

McGregor (1994) concibió también que el desarrollo humano es autogenerado y que avanza en un ambiente de confianza, retroalimentación y relaciones humanas auténticas. Consideraba que las ciencias sociales podrían contribuir eficazmente al progreso de la administración al lado humano de la empresa.

De este modo, la evolución del sentido de lo humano aumenta y se hace evidente que la administración no concuerda exactamente con la realidad a la que pretende aplicarse, por lo que se empieza a entender que la empresa se ha tratado como un universo aislado del resto de la sociedad y entonces la sociología comienza a demostrar que los hombres parecen producir mejor si son felices (Aktouf, 2001).

El sentido de lo humano en la administración empieza a tener relevancia para su gestión, ya que el buen trato hacia los seres humanos que forman parte de las instituciones permite que su motivación para el trabajo sea consagrada a la labor y, en esta lógica, la forma de sentir del trabajador lo beneficia a sí mismo como la organización a la cual pertenece (Cruz, Aktouf y Carvajal, 2003).

\section{El inicio en la gestión y su renovación}

En este apartado se retomarán los debates teóricos y sus praxis actúales sobre la renovación de la administración hacia el enfoque humanista y de gestión, lo cual remite directamente a los postulados de la escuela francesa y la Escuela de Altos estudios en Administración (HEC) de Montreal, Canadá, en las que se destacan los trabajos realizados por sus principales representantes: Omar Aktouf, de la escuela de Montreal, y Le Mouel, de la escuela francesa. Estos trabajos se retoman por la consistencia de su desarrollo teórico, la actualidad de sus análisis y, sobre todo, la perspectiva de ser humano que elaboran con la rigurosidad necesaria y que toma mayor fuerza como parte de la gestión humana en América Latina y Colombia. 
La propuesta de la escuela de Montreal, cuyos orígenes se remontan a la década de los años 60, implicó una apertura para entender la administración desde una perspectiva con rostro humano (Aktouf, 1989) y ubicada en el pensamiento administrativo renovador. Esta escuela contribuyó al debate acerca del futuro de las sociedades y la relación de los hombres consigo mismos y con su medio ambiente. Las posturas de esta escuela son asumidas de manera multidisciplinaria y su interés no es crear nuevas técnicas de gestión, sino darle un lugar a las ciencias de la vida. La perspectiva de las ciencias de la vida y la gestión es hoy en día reconocida como una disciplina científica en el campo del conocimiento administrativo, de modo que varios autores se refieren a ella como una apertura a repensar radicalmente la administración (Castro \& Lauriol, 1989).

Entre los postulados de la escuela de Montreal, se destacan las conclusiones que se presentaron en el coloquio realizado en Canadá en septiembre de 1990, cuyo tema central fue "El individuo en la organización. Las dimensiones olvidadas", las cuales están enmarcadas en la naturaleza genérica y singular del ser humano, su carácter activo y reflexivo, el uso de la palabra, la vida afectiva, la producción simbólica, la concepción espaciotemporal, la alteridad como proceso de construcción del individuo (Zapata, 1995; citado por López, 2007). Al respecto, Zapata (2005) plantea que el reconocimiento de estas dimensiones olvidadas del individuo en la organización, también llamadas las caras ocultas de la administración, da origen a una antropología de la organización, las cuales, según Jean-Chanlat (1982), se encuentran en cinco niveles estrechamente ligados: el individuo, la interacción, la organización, la sociedad y el mundo.

La propuesta de la escuela de Montreal, denominada gobierno de las personas, forma parte de las prácticas de gestión que permiten al dirigente comprender con precisión en cada situación las características específicas del contexto en el cual se encuentran los rasgos únicos de las personas con las cuales él debe entrar en relación.

Uno de los grandes representantes de la nueva visión renovadora de la administración y la gestión, que forma parte de la escuela de Montreal, es Omar Aktouf, quien ha formulado críticas importantes a la administración tradicional en su obra La administración entre tradición y renovación. En este trabajo el autor refuta lo que él denomina los abusos "de verdades" que pueden considerarse como sofismas que se han aceptado como verdades absolutas sin permitir cuestionamientos. Entre estas se encuentra la propiedad privada, considerada como un invento de Occidente sin ningún sustento histórico para derivar derechos en favor de los más ricos y en detrimento para la mayor parte de la población (Aktouf, 2001). 
Aktouf (2001; citado por Monroy, 2002) considera que otro sofisma derivado del anterior son los "derechos naturales" de quien detenta el poder conferido por la propiedad privada, para decidir y hacerse obedecer, privilegio que eventualmente le permite "eliminar" a los más débiles en su paso hacia la consolidación absoluta.

Estas verdades presentadas como sofismas constituyen una visión de túnel para los profesionales, pues se asume una verdad y se desdibuja su contexto, su dinámica y los rostros humanos que la configuran. Desde esta perspectiva se entiende a la gestión exclusivamente como un proceso a través del cual se planifica, dirige, controla y evalúa los recursos humanos, materiales y financieros para la consecución de objetivos

Por su parte, Le Mouel (1992), de la escuela francesa, plantea una fuerte crítica a la eficacia. Afirma que todo tipo de organización asume la eficacia en el marco de un sistema social y de conocimiento, y argumenta que las organizaciones sociales la aterrizan, particularmente en la escuela, bajo el sofisma de que "lo eficaz es verdadero, lo verdadero es justo, luego, lo eficaz es justo” (Le Mouel, 1992). Esto lleva a pensar que la eficacia se justifica como una transición para alcanzar un objetivo final, en el cual se legitima la idea de dejar a un lado el desarrollo de los valores — que cada vez van perdiendo más fuerza—, cuando en realidad se podría recuperar su verdadero sentido.

Los aportes de estos dos postulados coinciden en señalar la importancia que tiene otorgar a la educación la tarea de generar procesos de formación en gestión y reconstruir su sentido y praxis para atribuirle un verdadero sentido renovador. Para esto es importante reconocer los estudios adelantados por Benno Sander (1998) con respecto a las gestiones educativas y su desarrollo en América Latina.

Sander (1994) plantea que la gestión educativa ha estado en estrecha relación con la pedagogía desde su desarrollo. Desde este planteamiento, el autor destaca cinco etapas que corresponden a los enfoques conceptuales y analíticos mencionados previamente y que se profundizan en este apartado por su pertinencia para lograr una mejor comprensión de la gestión en la educación. Estos son: enfoque jurídico, tecnocrático, conductista, desarrollista, sociológico y el enfoque cultural pluridimensional (tabla 1). 
Tabla 1. Enfoques históricos de la gestión educativa en América Latina

Enfoque jurídico (desde el periodo colonial hasta las primeras décadas del siglo XX)

Características generales

- Normativo y vinculado a la tradición del derecho administrativo romano.

- Influencia europea en la cultura y la educación latinoamericana.

- Preferencia por el pensamiento deductivo.

- Pocos desarrollos teóricos y escasa sistematización del conocimiento en la administración.

- El cristianismo apoya la fuerza normativa y la lógica deductiva.

- En las primeras décadas del siglo XX se consolidaron las perspectivas tecno-burocráticas de la escuela clásica de administración.

\section{Desarrollo en la gestión educativa}

El positivismo se manifestó en la gestión educativa de Latinoamérica a través de la adopción de las perspectivas conductistas y funcionalistas de administración.

\section{Enfoque tecnocrático (primeras décadas del siglo XX)}

- La administración se inspiró en los principios de la escuela clásica de administración con sus movimientos científico, burocrático y general.

- Desarrolla la tecnocracia como sistema de organización.

- Preocupación por la adopción de soluciones racionales para resolver problemas organizacionales y administrativos.

- Las consideraciones políticas, los aspectos humanos y los valores éticos generalmente ocupaban un lugar secundario.

- Los principales autores fueron: Fayol en Francia; Taylor y sus seguidores en los Estados Unidos, y Weber en la fase reformista y de las etapas posteriores.

- Se asume un modelo máquina preocupado con la economía, la productividad y la eficiencia.

- Reducida atención a la influencia de los factores económicos, políticos y culturales en la vida de las organizaciones.
- Enfatiza la adopción de perspectivas y soluciones organizacionales desde el pragmatismo instrumental.

- Plantea que la pedagogía debía ofrecer soluciones técnicas para resolver racionalmente los problemas reales que afrontaba la gestión educativa. Sin embargo, la fuerza de la tradición del derecho administrativo romano continuó vigente en América Latina.

- En los años treinta se dio los primeros intentos de sistematización de las teorías organizacionales y administrativas de educación y los primeros ensayos de gestión educativa en América Latina.

\section{Enfoque conductista (a partir de la década de los años cuarenta)}

- Rescata la dimensión humana de la administración.

- Surge el movimiento psicosociológico de las relaciones humanas.

- Toma importancia el trabajo sobre el proceso de toma de decisiones y la conducta organizacional y administrativa.
- La dimensión subjetiva de la conducta humana está presente en la gestión educativa desde hace dos siglos, debido a la vinculación de la psicología con la pedagogía en los estudios de educación. 
Enfoque conductista (a partir de la década de los años cuarenta)

- La intersección teórica entre la psicología y la sociología dio origen, posteriormente, a la psicología social de la educación, que alimentó los estudios de gestión educativa por más de medio siglo.

- En la crisis mundial de la educación, se popularizó la utilización de la teoría de los sistemas como instrumento analítico para estudiar la situación y el funcionamiento de los sistemas educativos.
- Surge la perspectiva fenomenológica que concibe la gestión educativa como acto pedagógico en vez de definirla como acto empresarial o práctica comercial, pero no ha tenido la suficiente fuerza en América Latina.

\section{Enfoque desarrollista (posguerra)}

- De naturaleza modernizadora.

- Es el resultado de un conjunto de factores: la exposición internacional de los investigadores y ejecutivos norteamericanos durante la Segunda Guerra Mundial y la necesidad de organizar y administrar los servicios de asistencia técnica y ayuda financiera en la etapa de la posguerra.

- Proponen la adopción de una perspectiva de administración dedicada específicamente a la gestión de los programas de desarrollo para lograr sus objetivos económicos y sociales.

- Unesco, la OEA y la Cepal, consagran, en el ámbito político, el papel de la educación como factor de desarrollo económico, como instrumento de progreso técnico y como medio de selección y ascenso social.

- Encuentran en la tecnología el nuevo instrumento modernizador de la educación y la sociedad para América Latina.

- En la década de los ańos setenta la inversión en la educación latinoamericana no pagó los dividendos esperados en términos de crecimiento económico y progreso tecnológico, incluso en el desarrollo humano, calidad de vida y de equidad social. El optimismo pedagógico de las décadas anteriores entró en crisis.
- Se fortalece "la educación para el crecimiento económico".

- El sistema educativo deja de ser concebido como palanca y motor de cambio, y el optimismo pedagógico que concebía a la educación y al maestro como fuentes de progreso se ha transformado en pesimismo y desilusión.

- Se imponía así la necesidad de reevaluar el papel y la relevancia de la educación y la gestión educativa en América Latina.

- Las evaluaciones críticas revelaron que, entre otras conclusiones, el valor económico es una dimensión importante pero no suficiente de la gestión educativa.

Enfoque sociológico (años 80 - actualidad)

- Presenta las contribuciones conceptuales y analíticas de las ciencias sociales aplicadas de los autores latinoamericanos de las últimas décadas

- Mantiene preocupación por la concepción de teorías sociológicas y soluciones educativas para satisfacer las necesidades y aspiraciones de la sociedad latinoamericana.
- La gestión educativa desempeña una función esencialmente política, sociológica y antropológica

- Creación de asociaciones profesionales y académicas en distintos países de América Latina y del Caribe. 


\section{Enfoque sociológico (años 80 - actualidad)}

- El protagonista más influyente del pensamiento crítico latinoamericano de la época fue Freire.

- La teoría de la dependencia representa una contribución históricamente importante (Berger).

- Los nuevos pensadores enfrentan el desafío de concebir perspectivas de administración basadas en los valores culturales y políticos de la sociedad latinoamericana y de sus instituciones sociales.

- Es necesario el estudio de carácter interdisciplinario en la administración de la educación.
- Desarrollo de cursos de posgrado en educación, particularmente en el campo del planeamiento y la gestión educativa, como resultado de esfuerzos nacionales y de la cooperación técnica internacional

Fuente: Original de los autores con base en Sander (1996b).

La perspectiva histórica de la gestión que presenta Sander destaca los avances para el enriquecimiento epistemológico de la gestión y los enfoques desde los cuales se puede abordar. Sin embargo, a pesar de que se avanza desde un enfoque sociológico de comprensión de la gestión, en muchos contextos organizativos, empresariales y educativos prima la gestión con enfoque jurídico y tecnocrático, la cual desdibuja las perspectivas humanistas como camino para la renovación.

En Colombia hay avances importantes por parte de algunas instituciones de educación superior (IES) como la Universidad del Valle, la Universidad Eafit y la Universidad Pedagógica Nacional, y sus grupos de investigación sobre el tema, que le apuestan a desarrollar líneas de trabajo curriculares en las que se aborda la gestión desde el enfoque humanista. Esto ha tomado fuerza y requiere continuidad en la generación de líneas de comprensión, espacios de debate y formación crítica en la administración, educación y pedagogía, incluso con la participación de otras disciplinas que concuerdan con los principios aquí planteados.

\section{Perspectiva renovadora de la gestión en la educación}

Para abordar la perspectiva renovadora de la gestión educativa, en este apartado se retoman los planteamientos de Sander y Casassus. Estos autores desarrollaron sus principales postulados desde una perspectiva humanista de la gestión en la educación y ambos hacen un llamado importante para comprender que la institución educativa, al ser un sistema abierto en el que intervienen diversos actores, 
debe estar dispuesta a la transformación de perspectivas y enfoques que se han utilizado y de los cuales es necesario aprender y avanzar.

Para este estudio, los planteamientos de estos autores se presentan a través de una matriz que permite identificar los elementos centrales de sus postulados. Asimismo, se analiza la relación y diferencias entre cada uno.

Benno Sander exige un enfoque interdisciplinario para explicar los dominios de las influencias económicas, políticas, culturales y pedagógicas en la organización y administración, por ello le apunta a estrategias que combinen calidad, política, excelencia académica, eficiencia organizativa y democratización de la educación.

El autor expresa que en la gestión educativa es indispensable que confluyan aspectos como democracia, participación, así como otros relacionados con la calidad de la educación, lo cual permite una construcción integradora y colectiva de la gestión. Asimismo, establece la necesidad de racionalidad y pertinencia en el proceso administrativo y pedagógico. Adicionalmente, plantea que en la administración de los servicios sociales, como los de educación, la permanente participación de los ciudadanos es el requisito indispensable para lograr elevados niveles de calidad con equidad (Sander, 1994).

Los principales postulados de este autor se presentan en la tabla 2.

Tabla 2. Principales postulados de Benno Sander

\section{Obras de referencia:}

- Educación, administración y calidad de vida (1990)

- Gestión educativa y calidad de vida (1994)

- Nuevas tendencias en la gestión educativa: democracia y calidad (1996b)

\section{Enfoque}

\section{Definición \\ de gestión educativa}

Interdisciplinario y multidimensional: permite explicar los dominios de las influencias económicas, políticas, culturales y pedagógicas en la organización y administración de la educación en el hemisferio occidental.

- "La gestión en la educación no es una mera adaptación y apropiación de la administración a los procesos educativos, sino es un ámbito más de la educación en relación con la pedagogía y otras ciencias que allí intervienen, por lo que posee su propio cuerpo de conocimientos teóricos y prácticos históricamente construidos, en relación con la política y los sistemas educativos que de ella se derivan, así como de los desarrollo sociales y culturales" (Sander, 1990).

- "La escuela, al igual que la comunidad local, se deben autogobernar, conquistando mayores niveles de autonomía y descentralización administrativa" (Sander, 1996b).

- "Organizar las instituciones educativas y sus procesos administrativos y pedagógicos con racionalidad y pertinencia” (Sander, 1996b). 


\section{Obras de referencia:}

- Educación, administración y calidad de vida (1990)

- Gestión educativa y calidad de vida (1994)

- Nuevas tendencias en la gestión educativa: democracia y calidad (1996b)

$\begin{array}{ll}\begin{array}{l}\text { Definición } \\ \text { de gestión } \\ \text { educativa }\end{array} & \text { - "Es vista como un derecho y un deber de los integrantes de una comunidad } \\ & \text { democrática" (Sander, 1994). } \\ & \text { "Implica la deconstrucción y la reconstrucción de los conocimientos pedagó- } \\ & \text { gicos y administrativos" (Sander, 1996b). } \\ & \text { "Reconoce la necesidad de analizar y explicar los actos administrativos que } \\ & \text { ocurren en la escuela, la universidad y el sistema educativo como un todo, a } \\ & \text { la luz del concepto de calidad de vida humana" (Sander, 1994). } \\ & \text { "Estrategias en las que se combinan calidad, política, excelencia académica, } \\ & \text { eficiencia organizativa y democratización de la educación" (Sander, 1994). }\end{array}$

\section{Postulados que aporta a la comprensión de la gestión educativa}

\section{Principales críticas a la gestión}

\section{Retos para la gestión en la educación}

- "Construir una perspectiva democrática de gestión educativa como proceso de participación colectiva a la luz del concepto de calidad de vida” (énfasis agregado) (Sander, 1994).

- "Participación, concebida como derecho y deber de todos los ciudadanos que integran una sociedad democrática" (Sander, 1994).

- "[La] sociedad libre y equitativa basada en la calidad de vida humana implica, por parte de los actores sociales, la combinación correcta del interés individual con el compromiso social" (Sander, 1994).

- "En el campo de la sociología organizacional y la gestión educativa de las últimas décadas ha habido mayor preocupación por la denuncia y la crítica de la situación social y educativa, que por la definición de posibilidades concretas de acción humana para elevar el nivel de calidad de vida y de educación" (Sander, 1994).

- "Todavía no se ha consolidado una perspectiva comprensiva que sirva de guía para la investigación y la práctica de la gestión educativa” (Sander, 1994).

- "Los principios normativos y universales de los paradigmas tradicionales de administración deben dar lugar a la adaptabilidad y la flexibilidad en función de características concretas" (Sander, 1996b).

- "Desconstrucción y la reconstrucción de los conocimientos pedagógicos y administrativos acumulados en el curso de la historia de la educación latinoamericana. En realidad, los modelos históricos de gestión escolar y universitaria" (1996b).

- "Es necesario defender la tesis de la especificidad de la administración de la educación como campo teórico y praxiológico. De no ser así, corremos el riesgo de descaracterizar la misión de la educación y el objetivo primordial de la escuela y de la universidad en la sociedad moderna" (Sander, 1996b).

- "La acción individual debe incorporarse a la acción colectiva mediante una nueva ética de cooperación y participación ciudadana" (Sander, 1996b).

- "Una administración de la educación innovadora. Una administración construida y ejercida colectivamente. Una administración capaz de promover una educación con identidad. Una educación con equidad. Una educación de calidad para todos" (Sander, 1996b). 
Por su parte, el enfoque de Juan Casassus sobre las emociones es utilizado en el campo de la salud, la educación, la gestión y la terapia. En relación con esto último, fundó el Espacio Índigo, un centro cultural para el desarrollo del cuerpo, la mente y el espíritu. Es especialista regional en políticas, planificación y gestión educativas de la Oficina Regional de Educación para América Latina y el Caribe (OREALC/ Unesco) (Ebiblioteca, 2013).

Este investigador hace énfasis en que el desarrollo de la gestión debe estar centrado en la interacción de las personas, por eso el ser humano es el elemento crucial que hace posible el funcionamiento de las organizaciones.

Casassus (2000) asegura que la gestión en la educación inicia con los procesos de descentralización, en los cuales la actividad de gestión transita de la gestión del sistema en su conjunto, a la gestión de un sistema que está compuesto por distintas entidades y niveles de competencia de gestión.

Los principales planteamientos del autor se destacan en la tabla 3.

Tabla 3. Gestión en la educación de acuerdo con Juan Casassus

\section{Obras de referencia:}

- Problemas de la gestión educativa en América Latina (la tensión entre los paradigmas de tipo A y el tipo B) (Cassaus, 2000).

- Marcos conceptuales para el análisis de los cambios en la gestión de los sistemas educativos (Cassaus, 1999).

Enfoque

Definición de gestión educativa
Crítico: implica la relación entre gestión, educación y pedagogía y la necesidad de pensar de una manera nueva la posibilidad de organizar las escuelas y el sistema educativo desde el aprendizaje continuo, la generación de valores e interacciones que convergen en el desarrollo educativo

- "La gestión educativa busca aplicar los principios generales de la gestión al campo específico de la educación” (Cassaus, 2000).

- "La gestión educativa es una disciplina en gestación en la cual interactúan los planos de la teoría, los de la política y los de la pragmática” (Cassaus, 2000).

- "El concepto gestión, connota tanto las acciones de planificar como las de administrar" (Cassaus, 2000).

- "La gestión es la capacidad de generar y mantener conversaciones para la acción" (Cassaus, 1999).

- "En las distintas visiones de la gestión, está el tema del aprendizaje, el aprendizaje continuo, la generación de valores, la visión compartida, las interacciones, y las representaciones mentales. Estos temas mantienen directa relación con el desarrollo educativo" (Cassaus, 2000). 


\section{Obras de referencia:}

- Problemas de la gestión educativa en América Latina (la tensión entre los paradigmas de tipo A y el tipo B) (Cassaus, 2000).

- Marcos conceptuales para el análisis de los cambios en la gestión de los sistemas educativos (Cassaus, 1999).

\section{Definición de gestión educativa}

- La gestión educativa, debido a los cambios estructurales del sistema, ha pasado de ser una actividad exclusiva y propia de la cúspide del sistema (el ministerio central) a ser una actividad que ocurre en el conjunto del sistema (Cassaus, 2000).

- Desde el punto de vista organizacional, entonces la gestión se torna en el proceso de responder a las necesidades de los sujetos involucrados en el proceso con elementos tales como el compromiso y la satisfacción en el trabajo (Cassaus, 2000).

- La tradición de la gestión de la educación se inicia con los procesos de descentralización (Cassaus, 2000).

- Si la gestión tiende a definirse en relación con entidades administrativas, en la práctica es una actividad de relaciones entre sujetos (Cassaus, 2000).

- "Otro enfoque, es el lingüistico, el cual, focalizado en la comunicación, concibe que las personas se movilizan mediante compromisos adquiridos en la conversación. Así, la gestión es 'la capacidad de generar y mantener conversaciones para la acción" (Cassaus, 2000).

- "En la práctica, la perspectiva de gestión de calidad total en los sistemas educativos se orienta a mejorar los procesos mediante acciones tendientes, entre otras, a disminuir la burocracia, disminuir costos, mayor flexibilidad administrativa y operacional, aprendizaje continuo, aumento de productividad, creatividad en los procesos. Calidad total aparece entonces como la acción de revisión sistemática y continua de los procesos de trabajo, para identificar y eliminar los desperdicios. Esto requiere de la participación de los trabajadores hacia el mejoramiento continuo de sus labores como práctica laboral, mejorar la calidad de los procesos. Es la orientación para que se genere cero defectos en el proceso" (Cassaus, 2000).

- En el transcurso de esta trayectoria de concreción y flexibilización, se producen dos visiones que constituyen representaciones del contexto tanto interno como externo, en el cual operan las organizaciones. Estas dos reconstrucciones se presentan en el siguiente cuadro y las caracterizamos como visión de tipo A y visión de tipo B" (Cassaus, 2000):

- "El situarse en el tipo A implica usar ciertas técnicas de gestión que le sean afines, y no otras técnicas que no lo sean. Por ejemplo, el perfil real (no necesariamente el explicitado) que emerge de la política educativa, caracteriza la 'normalidad' en un país. Este perfil es un resultado de carácter homogéneo. Para ello es necesario utilizar técnicas de gestión que se focalicen en los resultados, la discreción de sus indicadores, su medición y su referencia a estándares".

- "Situarse en el modelo de tipo B, supone la aplicación de otras técnicas en la cuales se privilegia la diversidad, y se focaliza en los procesos y la calidad de las interacciones". 


\section{Obras de referencia:}

- Problemas de la gestión educativa en América Latina (la tensión entre los paradigmas de tipo A y el tipo B) (Cassaus, 2000).

- Marcos conceptuales para el análisis de los cambios en la gestión de los sistemas educativos (Cassaus, 1999).

\begin{tabular}{|c|c|}
\hline $\begin{array}{l}\text { Principales } \\
\text { críticas a la } \\
\text { gestión }\end{array}$ & $\begin{array}{l}\text { El Autor plantea los siguientes problemas de la gestión educativa en América } \\
\text { Latina: } \\
\text { - La ausencia de la dimensión “educación" en la gestión educativa (Cassaus, } \\
\text { 2000): en la formalización de la disciplina, la dimensión propia de la educa- } \\
\text { ción está ausente. } \\
\text { - En la última década, los conceptos que dominan la reflexión de la política } \\
\text { educativa tienen su raíz en la economía. Conceptos tales como la eficiencia, la } \\
\text { eficacia, la evaluación, productividad, competitividad, incentivos han copado } \\
\text { la literatura y el discurso de la política educativa (Cassaus, 2000). } \\
\text { - Tensión entre paradigmas concurrentes (Cassaus, 2000). En este paradigma, } \\
\text { lo importante es determinar con precisión el "producto" de la educación o } \\
\text { rendimiento del sistema, para así poder medirlo, determinar el ámbito y } \\
\text { niveles de "calidad", referirlo a "estándares" y a partir de ello, alinear procesos } \\
\text { para mejorar la productividad (eficiencia) y el producto (eficacia). } \\
\text { - Divergencia de los objetivos de la gestión desde la perspectiva de la escala } \\
\text { (Cassaus, 2000) en el que los intereses y objetivos de los niveles macro y los } \\
\text { de los niveles micro son divergentes, o dicho en lenguaje de gestión, presentan } \\
\text { problemas de alineamiento. } \\
\text { Desfase entre la formación en gestión y las demandas de la política educativa. } \\
\text { En la cultura de la tradición pedagógica no ha habido lugar para el desarrollo } \\
\text { de competencias de gestión (Cassaus, 2000). } \\
\text { Ausencia de investigación adecuada. En particular la investigación sobre los } \\
\text { modelos de gestión educativa (Cassaus, 2000). } \\
\text { Tensión que emerge de entre la visión autoritaria y la visión democrática de } \\
\text { la gestión. La gestión autoritaria está asociada a la gestión por resultados, } \\
\text { mientras que la democrática se asocia a la gestión de los recursos humanos } \\
\text { (Cassaus, 2000). }\end{array}$ \\
\hline $\begin{array}{l}\text { Retos para la } \\
\text { gestión en la } \\
\text { educación }\end{array}$ & $\begin{array}{l}\text { - Es necesario repensar la gestión educativa para que en ella la gestión no se } \\
\text { limite a la administración de los recursos, sino que abarque también el lide- } \\
\text { razgo pedagógico (Cassaus, 2000). } \\
\text { Es necesario profundizar el debate teórico. Se requiere mejorar el conocimiento } \\
\text { de las teorías, debatirlas y desarrollarlas (Cassaus, 2000). } \\
\text { - Nos encontramos en una situación en la cual existe evidencia generada en la } \\
\text { región según la cual se debería cuestionar con más fuerza la predominancia } \\
\text { del paradigma de tipo A (Cassaus, 2000). }\end{array}$ \\
\hline
\end{tabular}

Fuente: Original de los autores con base en Cassaus (2000).

Los planteamientos de estos autores han desarrollado una perspectiva renovadora de la gestión en la educación que ha tomado fuerza en los actuales debates sobre el tema en Colombia y Latinoamérica. Si bien cada uno elabora conceptos 
sobre la gestión en la educación, aún no se tiene un concepto concluyente, no porque no se pueda generar, sino por la dimensión compleja que representa esta nueva gestión. La condición humana es sin duda un entramado de dimensiones que aún se está explorando para comprender conductas, sistemas de valores, estrategias de interacción, entre otros aspectos que configuran el desarrollo y la evolución del ser humano.

Sin duda es un camino que está iniciando, pero realmente es necesario para abordar aspectos que contribuyan al mejoramiento de la calidad de vida desde la educación para la sociedad. La herramienta y el medio es la educación, así que desde los postulados y principios mencionados por estos autores vale la pena generar redes de conocimiento para estudiar las nuevas dimensiones de la gestión en la educación y nuevas líneas de investigación para su enriquecimiento. Por esto es necesario construir un desarrollo teórico desde la investigación y, en particular, aquella que busque en los educadores los aspectos necesarios para ello.

\section{Construcción pedagógica de la gestión, categorías de comprensión}

Finalmente, y con la intención de contribuir en la comprensión de la gestión en la educación, en este apartado se presentan esquemas de relaciones entre categorías emergentes por cada autor y desde la perspectiva humanista y sus enfoques sociales, renovadores y críticos de la administración y la gestión. De igual manera, con base en el análisis general de las relaciones entre las categorías emergentes de cada autor, se definieron categorías deductivas que se constituyen como nuevas categorías de comprensión de la gestión en la educación. Los esquemas se realizaron con base en un análisis de contenido utilizando el software de datos cualitativos Atlas Ti 6.0.

En primer lugar, en el análisis sobre los postulados de Sander se destaca la categoría de descentralización educativa como un reto de las instituciones para garantizar los espacios de participación y la configuración de nuevos escenarios de toma de decisiones en los que pueden intervenir diferentes actores. Al respecto, el autor señala que "la escuela, al igual que la comunidad local, se deben autogobernar, conquistando mayores niveles de autonomía y descentralización”" (Sander, 1996b). Incluso esta categoría es vista como un elemento importante que contribuye a mejorar la calidad de vida y el compromiso social, el cual establece en la gestión la posibilidad de darle el sentido transformador a la educación, pues posibi- 
lita cambios sociales para el desarrollo desde el reconocimiento de aspectos internos y externos de la formación (figura 1).

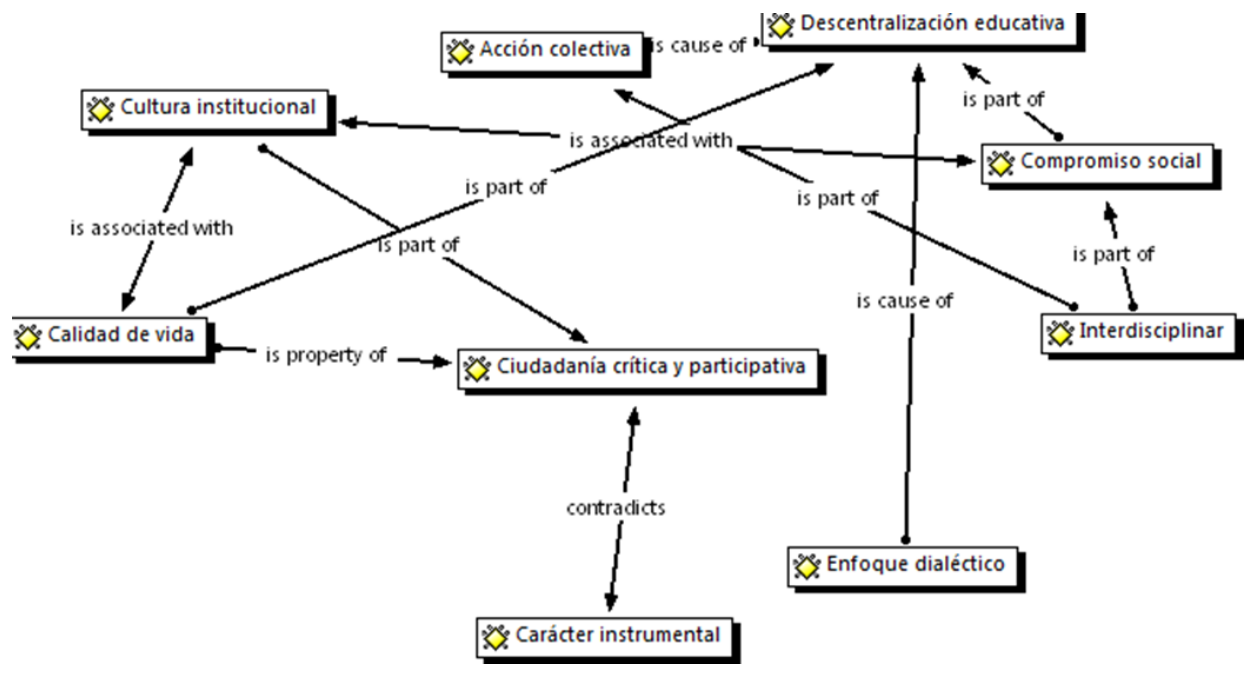

Figura 1. Postulados de Benno Sander.

Fuente: Original de los autores.

En segundo lugar, de acuerdo con las relaciones que se establecieron para los postulados desarrollados por Casassus, se puede identificar como principal categoría el enfoque dialéctico, el cual asume que la praxis entre el sujeto y la realidad es esencial para el proceso educativo de enseñanza-aprendizaje. Al respecto, el autor señala que "la gestión es la capacidad de generar y mantener conversaciones para la acción" (Casassus, 2000, p. 67). Esta categoría presenta más de dos relaciones con las demás categorías emergentes porque se asume como una causa del desarrollo de una ciudadanía crítica y participativa. Es decir, el proceso dialéctico solo puede ser asumido cuando tiene lugar un reconocimiento de los sujetos y su contexto con las dinámicas que surgen en este: "[Se] requiere de la participación de los trabajadores hacia el mejoramiento continuo de sus labores como práctica laboral, mejorar la calidad de los procesos. Es la orientación para que se genere cero defectos en el proceso" (Casassus, 2000, p. 32).

Adicionalmente, esta categoría está asociada con la cultura institucional y con la descentralización de la educación. Así, desde esta perspectiva se muestra la necesidad de incluir en la cultura institucional (entendida como todos los procesos administrativos y pedagógicos que intervienen en el contexto educativo) el sustento teórico y empírico que contribuye a la descentralización de la educación. 


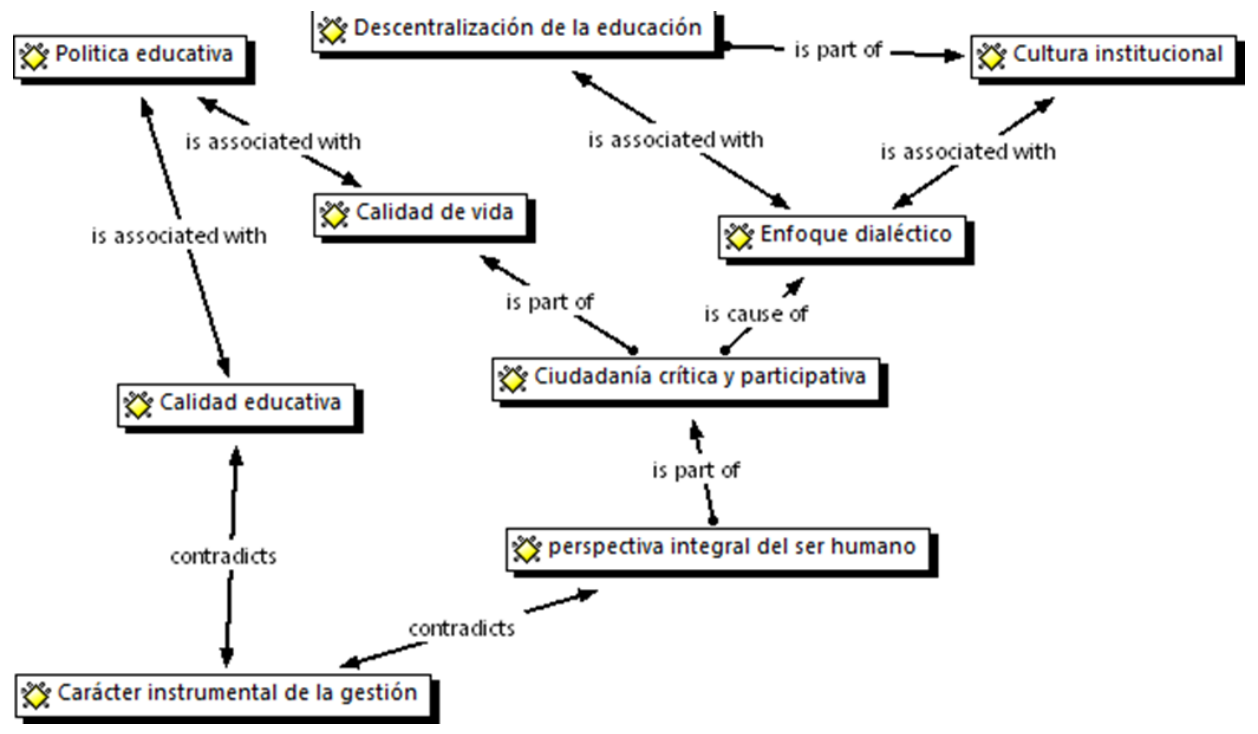

Figura 2. Postulados de Juan Casassus.

Fuente: Original de los autores.

\section{Reflexiones para el contexto de formación militar}

El proceso de formación militar está llamado a desarrollar nuevas formas de comprensión y análisis de los fenómenos sociales externos y de las dinámicas internas de enseñanza-aprendizaje. En este sentido, la perspectiva humanista de la gestión en la educación, de orden práctico, académico e investigativo, puede ser abordada de manera determinante en el contexto militar para avanzar en la consolidación de la formación integral que se proponen.

La formación integral que se aborda en el escenario militar puede ser asumida desde diferentes enfoques adaptados al contexto de cada país. Sin embargo, las perspectivas renovadoras sobre gestión en la educación toman un especial valor en la formación militar, toda vez que están en sintonía con las competencias profesionales que los centros de enseñanza militar buscan desarrollar en sus estudiantes para cumplir con las funciones y su campo ocupacional (Gutiérrez-Alban, Teneda \& Narváez, 2019).

Estas competencias profesionales se pueden identificar en diferentes líneas de acción, por ejemplo, aplicar los principios básicos de la pedagogía y la didáctica para desempeñarse como educador e instructor de la fracción a su cargo utilizando, cuando corresponda, herramientas de simulación, técnicas y procedimientos de empleo correspondientes (Riera, 2018). 
En este sentido, el componente que se acerca de manera más clara al escenario militar puede estar enfocado en la cultura institucional, que busca generar procesos académicos, administrativos e institucionales que se configuran como dinámicas e interacciones internas (entre miembros que constituyen el escenario de formación) y externas (con los entes que regulan las políticas educativas). La cultura institucional presenta un fuerte componente desde la política educativa, la cual afecta a los centros de formación porque pareciera muy rígida y los reta a posicionarse en espacios de encuentro y diálogo.

El llamado es entonces a analizar los lineamientos que plantean Benno Sander (1990, 1994, 1996b) y Juan Casassus $(1999,2000)$ a la luz de las dinámicas de formación militar, pues son principios que aportan en términos de mejoramiento de los procesos educativos, en la medida en que se plantea un abordaje de la gestión en la educación no como administración, sino en su relación con la educación, la pedagogía y la cultura. Esta perspectiva pone en el centro de la formación a los sujetos, que para este caso serían los militares, pues quiénes mejor que ellos para valorarlos.

La gestión educativa desde una perspectiva humanista busca ubicar al ser humano en el centro de los procesos que se abordan en las instituciones educativas. En este sentido, la formación militar tiene el importante reto de avanzar en la comprensión de este tema, pues trascender las visiones reduccionistas representa un avance importante para su proceso de transformación, de manera que le permita pasar de lo tradicional hacia lo renovador, respetando los ejes que fundamental y son esencia para los profesionales militares, pero reconociendo el carácter dinámico de la educación.

De acuerdo con las relaciones establecidas entre los autores seleccionados (Sander y Casassus) y sus principales postulados sobre la gestión en la educación, se identificaron cuatro categorías deductivas que permitieron tener una mayor comprensión teórica de la gestión en la educación. Desde esta perspectiva, la cultura institucional se comprende como el desarrollo de los procesos académicos, administrativos e institucionales que se configuran como dinámicas e interacciones internas (entre miembros que constituyen el escenario de formación) y externas (con los entes que regulan las políticas educativas).

\section{Financiación}

Esta investigación ha sido realizada gracias al financiamiento de la Escuela Militar de Cadetes "General José María Córdova” y la Escuela de Aviación del Ejército Nacional. Los autores declaran que no existe ningún potencial conflicto de interés relacionado con el capítulo. 


\section{Referencias}

Aktouf, O. (1989). Le managment entre tradition et renouvellement. Boucherville: Gaëtan Morin.

Aktouf, O. (2001). La administración: entre tradición y renovación. Cali: Artes Gráficas del Valle.

Alvarado, O. (1999). Gestión educativa: enfoques y procesos (1.a ed.). Lima: Fondo de Desarrollo Editorial Universidad de Lima.

Benno, S. (1990). Educación, administración y calidad de vida. Buenos Aires: Santillana.

Boeree, G. (2003). Teorías de la personalidad de Abraham Maslow [información en página web]. Recuperado de http://webspace.ship.edu/cgboer/maslowesp.html

Botero, C. (2009). Cinco tendencias de la gestión educativa. Revista Iberoamericana de Educación, 49 (2), 1-11. https://doi.org/10.35362/rie4922100

Cabrera, L. (2016). Principales enfoques y tendencias sobre la gestión en la educación en docentes de educación media y básica que cursan estudios de maestría, docencia e investigación universitaria en la Universidad Sergio Arboleda (Tesis de maestría Meritoria). Universidad Sergio Arboleda, Bogotá.

Casassus, J. (1990). Descentralización y desconcentración de los sistemas educativos en América Latina: fundamentos y dimensiones críticas. En Unesco Orealc, Proyecto Principal de Educación en América Latina y el Caribe (boletín 22) (pp. 7-16). Santiago de Chile: Unesco Orealc.

Casassus, J. (1999). Marcos conceptuales de la gestión educativa. En Autor, La gestión: en busca del sujeto. Documento presentado en el Seminario Internacional sobre Reformas de la Gestión de los Sistemas Educativos en la Década de los Noventa, Santiago de Chile, 1997.

Casassus, J. (2000). Problemas de la gestión educativa en América Latina (la tensión entre los paradigmas de tipo A y el tipo B). Santiago de Chile: Unesco.

Castro, J. L. \& Lauriol, J. (1989). L'excellence: du neuf et du vieux". Gestion. Revue Internationale de Gestion, 14 (4), 31-37.

Chanlat, A. (1982). La complémentarité dans la connaissance et l'action. Montreal: HEC, sans publier.

Cruz, F., Aktouf, O. \& Carvajal, R. (2003). El lado inhumano de las organizaciones. Cali: Universidad del Valle.

Ebiblioteca. (2013). Casassus Juan - Camino a la oscuridad [reseña del libro Camino a la Oscuridad, de J. Casassus [información en página web]. Recuperado de https://ebiblioteca.org/?/ver/89723

Escuela Militar de Cadetes "General José María Córdova” [ESMIC]. (2019). Perfil profesional del profesional en Ciencias Militares. Recuperado de https://www.esmic.edu.co/oferta_academica/ pregrados/facultad_ciencias_militares

Estrada, J. (2002). Configuraciones de política educativa neoliberal. En Viejos y nuevos caminos hacia la privatización de la educación pública. Política educativa y neoliberalismo. Bogotá, D. C.: Universidad Nacional de Colombia.

Fayol, H. (2000). Administración industrial y general. México: Herrero Hermanos.

Follet, M. P. (1965). Administración dinámica: colección de papeles de Mary Parker Follet. México: Herrero Hermanos.

Gutiérrez-Alban, A., Teneda Garcés, V. O. \& Narváez Ríos, M. M. (2019). La formación militar desde el enfoque por competencias: caso tecnología superior en ciencias militares. Revista de Ciencias de Seguridad y Defensa, 4 (8), 107-114. 
Lewin, K. (1951). Field theory in Social Science: Selected theoretical papers. Chicago: University of Chicago Press.

Le Mouël, J. (1992). Crítica de la eficacia. Ética, verdad y utopía de un mito contemporáneo. Buenos Aires: Paidós.

López, L. G. (2007). Humanismo y gestión humana: una perspectiva de interpretación para el trabajo social aplicado al campo laboral. Rev. Eleuthera, (1), 42-63. Recuperado de http://eleuthera. ucaldas.edu.co/downloads/Revista1_4.pdf

Mayo, E. (2004). The human problems of an industrial civilization. Londres: Routledge.

McGregor, D. (1994). El lado humano de las organizaciones. Bogotá: McGraw-Hill Interamericana.

Monroy, S. (2002). Los principios de Taylor y su aporte a la administración empresarial [Informe académico para la cátedra de Gestión]. Maestría en Administración, Universidad Nacional de Colombia, Bogotá.

Münch Galindo, L. (2007). Administración, escuelas, proceso administrativo, áreas funcionales y desarrollo emprendedor. México: Prentice Hall.

Riera, D. (2018). La cooperación en materia de Defensa en cuanto a la formación de oficiales de las Fuerzas Armadas de Argentina y Brasil (2008-2014) (Tesis de maestría). Centro de Estudios Interdisciplinarios, Universidad Nacional de Rosario, Argentina.

Sander, B. (1990). Educación, administración y calidad de vida: caminos alternativos del consenso y del conflicto. Buenos Aires: Santillana.

Sander, B. (1994). Gestión educativa y calidad de vida. La Educación: Revista Interamericana de Desarrollo Educativo, 118 (2), 237-264.

Sander, B. (1996a). Gestión educativa en América Latina: Construcción y reconstrucción del conocimiento. Buenos Aires: Troquel.

Sander, B. (1996b). Nuevas tendencias en la gestión educativa: democracia y calidad. Revista Interamericana de Desarrollo Educativo, 40 (123-125), 29-40.

Sander, B (2002). Nuevas tendencias en la gestión educativa: democracia y calidad [documento en página web]. Recuperado de https://pdfs.semanticscholar.org/8e5a/9d7f1fe8f2f67d0e77726c9d81a7bb2eb241.pdf?_ga=2.81990230.1519126882.1585853866-520944169.1585853866.

Stein, G. (1999). Peter F. Druker, Frederick W. Taylor y Mary Parker Follet: tres visiones complementarias del management. Empresa y Humanismo, 1 (2), 367-379.

Taylor, F. W. (2000). Principles of scientific management. Nueva York: Harper and Row Publishers.

Weber, M. (1947). The theory of social and economic organization. Nueva York: The Free Press.

Wolf, W. (1973). The impact of Kurt Lewin on management thought. Academy of Management, (1), 322-325. https://journals.aom.org/doi/pdf/10.5465/ambpp.1973.4981412

Zapata, A. (1995). La escuela de Montreal sobre humanismo y gestión. Cuaderno de Administración, (20), 3-11. https://doi.org/10.25100/cdea.v14i20.251 